\title{
Accurate simulation of backscattering spectra in the presence of sharp resonances
}

\author{
N.P. Barradas ${ }^{1,2}$, E. Alves ${ }^{1,2}$, C. Jeynes ${ }^{3}$, M. Tosaki ${ }^{4}$ \\ ${ }^{1}$ Instituto Tecnológico e Nuclear, Apartado 21, E.N. 10, 2686-953 Sacavém, Portugal \\ ${ }^{2}$ Centro de Física Nuclear da Universidade de Lisboa, Av. Prof. Gama Pinto 2, 1649-003 Lisboa, Portugal \\ ${ }^{3}$ University of Surrey Ion Beam Centre, Guildford GU2 7XH, England \\ ${ }^{4}$ Radioisotope Research Center, Kyoto University, Kyoto 606-8501, Japan
}

\section{This paper was published as:}

Nuclear Instruments and Methods in Physics Research B 247 (2006) 381-389;

http://dx.doi.org/10.1016/j.nimb.2006.02.004

In elastic backscattering spectrometry, the shape of the observed spectrum due to resonances in the nuclear scattering cross-section is influenced by many factors. If the energy spread of the beam before interaction is larger than the resonance width, then a simple convolution with the energy spread on exit and with the detection system resolution will lead to a calculated spectrum with a resonance much sharper than the observed signal. Also, the yield from a thin layer will not be calculated accurately. We have developed an algorithm for the accurate simulation of backscattering spectra in the presence of sharp resonances. Albeit approximate, the algorithm leads to dramatic improvements in the quality and accuracy of the simulations. It is simple to implement and leads to only small increases of the calculation time, being thus suitable for routine data analysis. We show different experimental examples, including samples with roughness and porosity.

PACS: 82.80.Yc, 07.05.Kf

Keywords: Elastic backscattering; resonance simulation; NDF 


\section{Introduction}

Elastic (non-Rutherford) backscattering spectrometry (EBS) is often used to profile light elements due to the existence of enhanced nuclear elastic scattering cross sections, particularly at resonances. If the probing beam energy is at or slightly higher than the resonance energy, the resonance occurs at or near the surface; if the beam energy is higher than the resonance energy then the resonance occurs deeper in the sample. The energy spread of the beam before interaction leads to a broadening of the resonance signal, and we will show that it also affects the yield of thin layers.

This effect has been previously considered for nuclear reaction analysis (NRA) and included in the NRA codes SPACES [1,2,3] and, more recently, Flatus [4]. A detailed analysis of this effect for EBS spectra of protons in carbon has been presented and included in the code BS1 $[5,6,7]$. However, the calculation of a double integral (on depth of scattering and on ion energy before scattering) is required, as opposed to the standard calculation of RBS spectra [8], that involves only a single integral (on depth of scattering).

This leads not only to complicated algorithms which are difficult to implement, but also to calculations which are several orders of magnitude longer, and impractical for routine data analysis even with modern PCs. In practice, standard analysis codes such as RUMP [9], SIMNRA [10] or NDF [11,12] do not include this double integration, and simulations of a resonance signal are normally sharper than the data. Also, the yield of a buried layer is calculated inaccurately.

Here we present a method, implemented in NDF, where the cross section is correctly calculated, and where the average scattered beam energy after interaction is also correctly calculated. The approximation is thus to disregard the exact shape of the energy distribution after interaction. Note however that, if the energy spread on the way out is larger than the resonance width, it dominates the shape of the energy distribution of the beam when it leaves 
the sample, and thus the details of the energy distribution after scattering become less relevant.

The advantages are the simplicity of implementation, together with fast calculation times: the algorithm presented can be easily included in any standard simulation code without significant loss of speed.

Also, the effect of sample features such as roughness or porosity on the energy spread can also be included in a natural way, without extra cost in terms of complexity or calculation time.

We present examples of proton backscattering in $\mathrm{C}$ and $\mathrm{Si}$. Dramatic improvements in both the calculated shape and yield of the resonances are obtained.

\section{Calculations}

\subsection{General considerations}

The general formula to calculate the yield Y due to scattering in a layer of thickness $\mathrm{Nt}$ is

$\mathrm{Y}=\sigma \Omega \mathrm{QNt}$,

where $\sigma \equiv \sigma(E)$ is the cross section, $\Omega$ the solid angle subtended by the detector, and Q the beam fluence. $\mathrm{N}$ is the density of the layer, and $\mathrm{t}$ its thickness, so $\mathrm{Nt}$ is the areal density, the quantity to which IBA techniques are sensitive. At a given depth t, the beam energy before interaction follows a distribution $\Gamma_{0}^{\mathrm{t}}$ (normally assumed to be Gaussian), characterised by its average $E_{0}^{t}$, and spread $s_{0}^{t}$. Each beam ion has a different energy before scattering at that depth, leading to a different cross section and a different detected energy. In the presence of a 
sharp resonance in the cross section, even if $\Gamma_{0}^{\mathrm{t}}$ is Gaussian, the energy distribution after interaction will have a complex non-Gaussian shape, since it will include the shape of the cross section (see figure 3 of ref. [5]). The yield dY measured at energy $E_{\text {out }}$ due to scattering of particles with energy $\mathrm{E}$ at a layer with thickness $\mathrm{d}(\mathrm{Nt})$ will be

$\mathrm{dY}\left(\mathrm{E}_{\text {out }}\right)=\sigma(\mathrm{E}) \Omega \Gamma_{0}^{\mathrm{t}}\left(\mathrm{E}_{0}^{\mathrm{t}}-\mathrm{E}, \mathrm{s}_{0}^{\mathrm{t}}\right) \mathrm{Qd}(\mathrm{Nt})$,

with

$\mathrm{E}_{\text {out }} \equiv \mathrm{E}_{\text {out }}(\mathrm{E})=\mathrm{KE}-\Delta \mathrm{E}(\mathrm{E})$,

where $\mathrm{K}$ is the kinematic factor (thus $\mathrm{E}_{1}=\mathrm{KE}$ is the particle energy after scattering) and $\Delta \mathrm{E}$ is the energy lost by the particle on the way out, which depends on energy. In order to obtain the energy spectrum, in principle a double integral must be calculated: on depth of scattering and on ion energy before scattering. This also implies calculating, for each depth, different energy losses for each ion energy before interaction, according to eq. (3). This method leads to intricate computer implementations which are too slow to be used in routine data analysis. The only code so far developed for RBS that implements eq. (2), is to our knowledge [13] the BS1 code developed specifically for proton scattering off pure carbon targets [5].

If the cross section is constant or changes linearly with energy in a vicinity of $E_{0}^{t}$ (which can be defined e.g. as $\mathrm{E}_{0}^{\mathrm{t}} \pm 2 \mathrm{~s}_{0}^{\mathrm{t}}$, to include $95 \%$ of the beam particles), then

$\int \sigma(E) \Gamma_{0}^{t}\left(E_{0}^{t}-E, s_{0}^{t}\right) d E=\sigma\left(E_{0}^{t}\right)$ 
and the integration of eq. (2) on energy before interaction $E$ leads to

$\mathrm{dY}\left(\mathrm{E}_{\text {out }}\right)=\sigma\left(\mathrm{E}_{0}^{\mathrm{t}}\right) \Omega \mathrm{Q} \mathrm{d}(\mathrm{Nt})$

with

$\mathrm{E}_{\text {out }} \equiv \mathrm{E}_{\text {out }}\left(\mathrm{E}_{0}^{\mathrm{t}}\right)=\mathrm{K} \mathrm{E}_{0}^{\mathrm{t}}-\Delta \mathrm{E}\left(\mathrm{E}_{0}^{\mathrm{t}}\right)$,

where $\sigma\left(E_{0}^{t}\right)$ is the cross section for the average energy of the beam at depth $t$, and the term $\Delta \mathrm{E}\left(\mathrm{E}_{0}^{\mathrm{t}}\right)$ is the same for all particles scattered at depth $\mathrm{t}$. In practice, this is approximately the case for the Rutherford cross section, and data analysis codes such as RUMP [9], SIMNRA [10], or previous versions of NDF [12], implement eq. (5). One single integral, on depth of scattering, is necessary, and the energy loss can be calculated only once for each depth, according to eq. (6). Energy spread (straggling) is included by convoluting the yield as calculated with eq. (5) with the energy spread of the beam on exit.

However, if the cross section changes rapidly with energy in the vicinity of $E_{0}^{t}$, then eq. (4) is no longer valid. This happens at low beam energies due to the $1 / \mathrm{E}^{2}$ dependence of the Rutherford cross section, or, more importantly, in elastic backscattering, whenever there is a resonance in the cross section with width comparable to, or smaller than, the energy spread $\mathrm{s}_{0}^{\mathrm{t}}$. Standard analysis codes will lead to simulations increasingly inaccurate for resonances that take place deeper in the sample, where the energy spread is larger. In general, the shape of resonances calculated with eq. (5) will be narrower than the experimental data, if the energy spread before scattering is similar to or larger than the resonance width.

Finally, evaluated cross sections [14] were used for $\mathrm{Si}$ [15] and C [16,17]. The 
contributions of pulse pile-up [18] and double scattering [19] were calculated and included in the simulations.

\subsection{Calculation model}

Our goal is to reproduce accurately the shape of deep resonances, without actually performing the double integral calculation, leading to an algorithm suitable for routine data analysis. First, we note that the effects of a cross section that changes rapidly with energy, combined with an energy spread at scattering, are: 1) The total cross section for scattering at a given depth is different from the cross section for the average energy $E_{0}^{t}$ of the beam at that depth; 2) the energy distribution after scattering is not Gaussian; and 3) in particular, the average beam energy after scattering is not $\mathrm{KE}_{0}^{\mathrm{t}}$. Our approximation consists in ignoring point 2), that is, the exact shape of the energy distribution after scattering, and calculating points 1) and 3) accurately. We note that the effect discussed in this work is only ever important if the scattering at the resonance energy takes place sufficiently deep that energy straggling on the way in has an important role; but in that case, the exact shape of the energy distribution after scattering will be smeared out by the energy straggling on the way out. This is the crucial point that justifies the approximation made.

The effective scattering cross section at depth $\mathrm{t}, \sigma^{\mathrm{t}}$, must be integrated over all beam energies at that depth:

$$
\sigma^{\mathrm{t}}\left(\mathrm{E}_{0}^{\mathrm{t}}\right)=\int_{-\infty}^{+\infty} \Gamma_{0}^{\mathrm{t}}\left(\mathrm{E}-\mathrm{E}_{0}^{\mathrm{t}}, \mathrm{s}_{0}^{\mathrm{t}}\right) \sigma(\mathrm{E}) \mathrm{dE}
$$

In this way, while $\sigma(\mathrm{E})$ is the well-defined energy-dependent cross section function, $\sigma^{t}\left(E_{0}^{t}\right)$ is the total cross section at a given depth, that is, averaged at that depth over the 
energy distribution of the inciding particles, which depends on target composition and thickness. Consequently, $\sigma^{t}\left(E_{0}^{t}\right)$ is a well-defined quantity that depends on target composition and thickness as well, and must be recalculated each time that the target structure changes during a fit or iterative calculation. Note the formal similarity between eqs. (7) and (4). In previous analysis codes the integral in eq. (4) was never calculated, and $\sigma\left(E_{0}^{t}\right)$ was directly taken. In the algorithm now developed, $\sigma^{t}\left(E_{0}^{t}\right)$ must be explicitly calculated as the integral in the right-hand side of eq. (7).

In a similar way, the average beam energy $E_{1}^{t}$ after scattering at depth $t$ is:

$\mathrm{E}_{1}^{\mathrm{t}}\left(\mathrm{E}_{0}^{\mathrm{t}}\right)=\int_{-\infty}^{+\infty} \mathrm{E}_{1}(\mathrm{E}) \Gamma_{0}^{\mathrm{t}}\left(\mathrm{E}-\mathrm{E}_{0}^{\mathrm{t}}, \mathrm{s}_{0}^{\mathrm{t}}\right) \sigma(\mathrm{E}) \mathrm{dE} / \int_{-\infty}^{+\infty} \Gamma_{0}^{\mathrm{t}}\left(\mathrm{E}-\mathrm{E}_{0}^{\mathrm{t}}, \mathrm{s}_{0}^{\mathrm{t}}\right) \sigma(\mathrm{E}) \mathrm{dE}$,

where, for elastic backscattering, $E_{1}=K E$. Note that the eqs. (7) and (8) must be calculated for each depth of scattering, that is, for each internal sublayer. This increases the calculation time by about $30 \%$, which is not only acceptable, in practice it is not noticeable since the calculation of one spectrum takes a fraction of a second in a modern PC. Thus, the algorithm developed here is suitable for routine data analysis, and is now integrated in the NDF code $[11,12]$. By default, the "resonance effect" is calculated whenever there is a non-Rutherford cross section. It is a user option otherwise, recommended only if higher accuracy of the calculation of the yield at low energies is required.

We show in Figure 1 the cross section $\sigma\left(E_{0}^{t}\right)$ for the ${ }^{12} C(p, p){ }^{12} C$ at a $160^{\circ}$ scattering angle, and the effective cross section for normal incidence in a pure $\mathrm{C}$ target, for different beam energies. The higher the beam energy, the longer the beam path required to reach the resonance energy at $1734 \mathrm{keV}$, and the higher the energy spread. This leads to a broadening of the effective cross section curve, larger for higher initial beam energy. Note that for an initial 
beam energy of $2.2 \mathrm{MeV}$, scattering at $1675 \mathrm{keV}$ leads to an average energy on exit of $0 \mathrm{keV}$, and thus the calculation stops.

We show in Figure $2 E_{1}^{t}-E_{1}$, that is, the difference in average energy after interaction due to the resonance effect, for the same conditions as in Figure 1. Changes around $10 \mathrm{keV}$ are attained, which is a large value with visible effects in the calculation. In the vicinity of the resonance, the energy after interaction values tend to become closer to the energy after interaction for scattering at the resonance energy. That is, there is an energy concentration effect around the resonance that makes the resonance signal sharper. This effect is in the opposite direction of the previous one, but is smaller and thus the global effect still is a broadening of the resonance signal.

In order to validate the approximations in our model, we compared the results obtained with those previously presented in ref. [5], where double integral calculations using eqs. (2) and (3) were done. The sample was dense HOPG graphite, measured with 5.05, 5.5 and 6.0 $\mathrm{MeV}$ protons at normal incidence and detected at $179.2^{\circ}$. Resonances $\mathrm{P} 1, \mathrm{P} 2$ and $\mathrm{P} 3$, at energies 4.8, 5.37 and $5.89 \mathrm{MeV}$ are observed. One limitation of the code BS1, used to perform the double integral calculations [5], was that it supports only Bohr straggling (including a multiplicative factor, if required). Other contributions to energy spread (such as the Tschalär effect $[20,21,22]$ which describes the way that straggling cumulates with depth) are not included in the BS1 model. In order to be able to compare the results, we now also used only Bohr straggling (but including the Tschalär effect, which is deeply imbedded in the straggling implementation). The results are shown in Figure 3. It is clear that, in general, the differences between the simulations with the full model (eqs. (2) and (3)) and with the approximation now developed (eqs. (5) and (6)) are smaller than the differences between the simulations and the data. The largest difference in calculated shape is observed for resonance $\mathrm{P} 1$ at a $6.0 \mathrm{MeV}$ beam energy, which is buried very deeply. 


\subsection{Energy spread, roughness, and inclusions}

Energy spread is calculated with the computer code DEPTH [23,24], which does state of the art calculations. It includes the effect of angular and energy spread of the beam, geometrical spread caused by the finite beam spot and detector aperture, Bohr straggling including the Chu correction, and multiple scattering calculated with the models developed by Amsel and co-workers [25]. DEPTH has been validated within 10\% for several systems, including $\mathrm{Si}$ [26], Si/Ge [27], and Co/Re [28]. Deviations up to 30\% have been observed for heavy ion ERDA using a $154 \mathrm{MeV}{ }^{197} \mathrm{Au}$ beam on $\mathrm{Al}$ and $\mathrm{Co}$ thin films [29].

Sample effects can lead to additional contributions to the energy spread of the beam, at a given depth. These can be added, in quadrature if Gaussian energy distributions are assumed, to the energy spread as calculated with DEPTH. For instance, inhomogeneity in the thickness of a layer leads to a spread in the energy lost by the beam in that layer. Also, if inclusions (or pores) are present, the stopping power is different from the stopping power in the matrix. Each beam particle can cross a different number of inclusions, resulting in additional spread in their energy. Roughness and porosity can lead to other effects in the data besides increased energy spread. However, given well-defined assumptions and limits of validity, the additional energy spread will be the most important effect, sufficient to describe the data.

We have previously developed analytical models to calculate the contribution to energy spread of different types of roughness, such as substrate surface roughness, layer corrugation, inhomogeneous layer thickness, and roughness/interdiffusion in multilayers [28,30,31]. We used this approach to study roughness in a number of different systems [30-33]. Stoquert and Szörenyi developed a model to calculate the contribution to energy spread of spherical and columnar pores and inclusions, and applied it to several cases [34]. They calculated only the 
energy spread, without actually calculating theoretical RBS spectra and fitting it to the data. We extended this approach to include cylindrical voids and inclusions, and included it in NDF, such that energy spectra can be calculated easily and fast. Channelling can also lead to resonance broadening [35].

\section{Experimental details}

Different pure carbon samples were measured: glassy carbon, which does not have pores in spite of its low density; high density pyrolytic graphite; and a graphite rod with $\rho=1.79 \mathrm{~g} / \mathrm{cm}^{3}$. The surface roughness was measured with a Sloan DEKTAK 3030ST profilometer with which several $1 \mathrm{~mm}$ long scans were made in different parts of the sample and in different directions. The average and standard deviation of the results were taken as the roughness and respective error. A crystalline (100) Si sample was also measured. RBS experiments were done on these samples using a $\mathrm{H}^{+}$beam at different energies, using the Van de Graaff accelerator at ITN. The scattering angle was either $160^{\circ}$ in the Cornell geometry or $140^{\circ}$ in the IBM geometry. The angle of incidence $\theta$ between the beam and the normal to the sample surface was varied between $0^{\circ}$ and $75^{\circ}$. In some cases, experiments were made before and after polishing the sample surface.

A transmission sample of nickel on carbon was made by glueing a $4 \mu \mathrm{m}$ thick Ni foil to the top of an aluminium frame about $1 \mathrm{~mm}$ thick, and a $0.9 \mu \mathrm{m}$ mylar foil to the bottom of the frame (both $\mathrm{Ni}$ and mylar as received from Goodfellow Metals). Backscattering proton spectra were collected from a detector at a scattering angle of $149.2^{\circ}$. The $\mathrm{H}^{+}$beam struck the sample at normal incidence, and the transmitted beam continued down a long beam line. No scattered beam from the beam stop reached the detector. We used various proton energies between 1800-2600 keV from the 2MV Tandetron at Surrey [36]. 


\section{Results}

The results obtained for 1.8 and $2 \mathrm{MeV}$ protons in a glassy carbon sample are shown in Figure 4. The scattering angle was $160^{\circ}$ and normal incidence was used. The $35(10) \mathrm{nm}$ surface roughness are included in the calculations, but have almost no effect. For a $1.8 \mathrm{MeV}$ beam energy, the energy spread at the resonance energy is still small, and the calculations with and without including the effect are only slightly different. However, for an initial 2 $\mathrm{MeV}$ beam energy, the resonance takes place deeper in the sample and the effect is more noticeable. The energy spread before interaction (FWHM) calculated with DEPTH is 38 and $77 \mathrm{keV}$ for 1.8 and $2 \mathrm{MeV}$, respectively, compared with the FWHM of the resonance, around $45 \mathrm{keV}$. It is interesting to note that, if only the effective cross section was calculated, and the change in the average beam energy after interaction was ignored, the resulting calculated resonance shape in the EBS spectrum would be much too broad.

The results obtained at $140^{\circ}$ scattering angle and $5^{\circ}$ tilt angle with $2.0 \mathrm{MeV}$ protons on high density pyrolitic graphite are shown in Figure 5. The surface roughness was 630(200) nm before polishing and 36(10) after, corresponding to a energy spread (FWHM) of 35 and 2 $\mathrm{keV}$, respectively. This compares to the calculated $32 \mathrm{keV}$ energy spread due to straggling. Therefore, while after polishing the energy spread before interaction is mainly due to straggling alone, before polishing the contribution due to roughness cannot be ignored. It is clear that, once the resonance effect is taken into account, including the energy spread due both to straggling and to roughness leads to an excellent agreement with the data. One should note that, adjusting the roughness value within its experimental uncertainty would further improve the simulations.

We analysed a porous graphite with $\rho=1.79 \mathrm{~g} / \mathrm{cm}^{3}$, that is, with a 20 vol.\% void fraction. The measured surface roughness was $335(180) \mathrm{nm}$, corresponding to a $18 \mathrm{keV}$ energy spread. The results for a $2 \mathrm{MeV}$ proton beam detected at $140^{\circ}$ with a $5^{\circ}$ tilt angle are 
shown in Figure 6. It is clear that the porosity must be included to obtain a reasonable simulation. Nevertheless, the shape of the experimental data is not as well reproduced as in the previous examples. On the one hand, the porosity model considers all voids to be of the same shape and size; a distribution in any of these parameters would lead to further energy spread. On the other hand, the contribution to energy spread due to the sample (roughness and porosity) is very large in this case, and the approximate models developed may be close to their limit of validity [31]. Nevertheless, the agreement of the simulation and the data must be considered quite good, particularly if compared with the simulations that do not take the resonance effect into account.

We show in Figure 7 data for the same glassy carbon sample as in Figure 4, for $2 \mathrm{MeV}$ protons. However, the scattering angle was $160^{\circ}$ in the Cornell geometry, with incidence angle from $0^{\circ}$ (normal incidence) to $75^{\circ}$ (grazing incidence). In this way, we can test the effect of changing energy spread due to the increased multiple scattering at more grazing angles. It is clear that for larger tilt angles, the simulations become increasingly worse. While the width of the resonances continues to be well calculated, their area and peak position is not. The calculated area becomes larger than the observed one, and while the observed peak clearly shifts to lower energies for higher tilt angles, the calculated peak remains approximately in the same position, or even shifts slightly to higher energies.

From a theoretical point of view, it is not well understood why the measured peak maximum should shift to lower energies. One possibility is that, considering scattering at a given depth, angular spread will lead to a spread of outward trajectories with different path lengths, with different total energy loss for each one. Approximate calculations show that, for grazing incidence, integration of the energy loss over the angular spread leads to an average energy loss larger than the energy loss for the straight trajectory, and that the effect is larger for higher tilt angles. We tried to include this effect in NDF using the angular spread 
calculated with DEPTH, but the quantitative results do not match the data; a change in energy loss a factor of 3 to 5 larger than the calculated change due to this effect would be required. One must note that, anyway, the multiple scattering theory behind DEPTH fails at grazing angle, due to the assumption of symmetrical scattering in respect to the trajectory direction $[25]$.

The observed decrease in the area of the resonance signal at high angles of incidence is also not well understood. Effects due to multiple scattering could also play a role, since angular spread on the way in and on the way out, combined, lead to a spread in the scattering angle, with possible effects in the cross section. Approximate calculations implemented in NDF using the angular spreads calculated with DEPTH added in quadrature to obtain the scattering angle spread (which is certainly not valid), and using evaluated cross sections for a range of angles, lead to a small correction only, quantitatively very far from the experiment.

We show in Figure 8 results for a c-Si sample, obtained with a $2 \mathrm{MeV}$ proton beam detected at $160^{\circ}$ in the Cornell geometry. Rotating pseudo-random spectra were collected at different angles of incidence. Again, while for near-normal incidence the simulations are almost perfect, for grazing angle strong deviations are observed. In particular, and similarly to what was observed with carbon, the observed peak is at lower energies than the calculated peak.

We show in Figure 9 three of the many spectra obtained from the Mylar $1 \mu \mathrm{m} / \mathrm{Ni} 4.2$ $\mu \mathrm{m}$ sample. The proton beam first crossed the Ni film leading to a high energy spread at the Mylar. The observed oxygen concentration was substoichiometric, indicating loss of O during the experiment. A small further loss of $\mathrm{O}$ was observed during the series of experiments at different beam energies in the same spot. As the energy spread arises almost entirely in the $\mathrm{Ni}$ film, with the contribution to energy spread due to the $\mathrm{O}$ in the Mylar being extremely small, this does not affect the results. 
The spectra were analysed taking the $\approx 0.36 \mu \mathrm{m}$ roughness of the Ni film into account with the thickness inhomogeneity model previously developed by us [30]. In order to check whether this leads to a correct calculation of the $\mathrm{C}$ yield, we also made a superposition of spectra calculated for many different Ni layer thickness values, with a Gaussian distribution. The $\mathrm{C}$ yield was in all cases the same within $2 \%$, validating once again the roughness algorithms in NDF, in what is an extremely demanding test. Note that the position of the simulated $\mathrm{C}$ peak does not match the data exactly. The small difference could be due to $\mathrm{a} \approx 2 \%$ inaccuracy in the stopping power data base.

First we analysed the spectrum collected at $2577 \mathrm{keV}$. For this initial energy the beam particles reach the Mylar layer with an energy distribution still within the plateau of the cross section, and as expected the $\mathrm{C}$ yields obtained with and without taking the effect into account are practically the same, and thus this spectrum serves as reference. We fitted the Mylar thickness in order to reproduce the observed C yield. Then, for that Mylar thickness, we calculated the spectra, with and without the resonance effect, for all other beam energies.

It is clear from Figure 9 that if the resonance effect is not considered, large quantitative and qualitative errors can arise. For $2066 \mathrm{keV}$, the average beam energy at the Mylar is close to the resonance energy of $1734 \mathrm{keV}$, and the $\mathrm{C}$ yield is at the maximum; without the resonance effect, the calculated yield is too large by 40 to $50 \%$, which, in resonant depth profiling, would lead to a corresponding error in the $\mathrm{C}$ concentration determined. On the other hand, at $2025 \mathrm{keV}$, where the beam reaches the Mylar layer with an energy smaller than the resonance energy, the calculated $\mathrm{C}$ yield is one order of magnitude too small. In fact, it is not possible to obtain a good simulation if the resonance effect is not taken into account.

On the other hand, once the resonance is considered, all spectra are well simulated. We show in Figure 10 the integral of the $\mathrm{C}$ peak for all the beam energies. It is clear that an extraordinarily good agreement is obtained. Note that this validates not only the algorithm 
now presented, but also the energy spread calculations, including the contribution of roughness. On the other hand, if the resonance effect is not taken into account, agreement is obtained only for beam energies at the Mylar layer well away from the resonance. As resonant depth profiling is always made at the resonant energy in order to maximise the measured yield, it is clear that, for sufficiently deep layers, very large errors can be committed.

\section{Conclusions}

We have presented a simple approximate algorithm that leads to significant improvements in the simulation of resonances in elastic backscattering whenever the energy spread of the beam before scattering is similar to, or larger than, the width of the resonance. This is normally the case for so-called buried resonances, when the initial beam energy is much larger than the resonance energy. In this case, significant energy straggling has occurred by the time that the beam particles have lost enough energy to reach the resonance energy.

The resonance effect leads to a change of the total cross section for scattering at a given depth, and to a change in the shape and average value of the energy distribution of the beam after scattering. The approximation made by us consists in disregarding the exact shape of the energy distribution after scattering, while calculating correctly the cross section and the average beam energy after scattering. The strength of the approximation lies in the fact that energy straggling on the way out leads to a smearing of any features of the energy distribution.

Roughness, porosity, and inclusions (such as quantum dots or nanoparticles) can be included in the simulations, by calculating their contribution to the energy spread of the beam via analytical models.

The algorithm can be easily implemented in any standard data analysis code, without large increases in calculation time. We have included it in the NDF v8.2 [37] code, for RBS 
and ERDA whenever a non-Rutherford cross section is present, and for NRA. As an user option, it can also be used for Rutherford cross sections, which may be important if an accurate calculation of the yield at low energies is required.

We showed examples of proton backscattering off $\mathrm{C}$ and $\mathrm{Si}$ targets. In most cases, the agreement between the simulations and the data is excellent, even in the presence of roughness and porosity. In all cases where the energy spread is large, dramatic improvements in the simulations are obtained. In particular, we show that the calculation of the yield of a buried layer containing C must include this effect. Otherwise, errors around 30 or $40 \%$ can be made at the resonance maximum, while outside the maximum the calculated yield can be wrong by one order of magnitude.

At grazing angles of incidence, while the shape and width of deep resonances is well reproduced by the algorithm now presented, their area and peak position are not. The reasons for this are still unclear, but could be related to the fact that current multiple scattering theory has problems at grazing angles.

\section{Acknowledgments}

The authors thank Dr. Alexander Gurbich for very useful discusions and for providing the cross sections used in this work, Drs. João Guilherme Correia, Ana Rita Ramos, António Gonçalves and Prof. José Carvalho Soares for providing test samples, Dr. Susana Freitas for use of the profilometer at INESC-MN, and Dr. Edit Szilágyi for discussions. NPB thanks Nick Dytlewski and the International Atomic Energy Agency for organising an exercise on Intercomparison and Evaluation of Software for Accelerator-Based Nuclear Techniques of Analysis, that led to this and other developments in NDF. 


\section{References}

[1] B. Maurel, G. Amsel, J.P. Nadai, Nucl. Instrum. Methods 197 (1982) 1.

[2] I. Vickridge, G. Amsel, Nucl. Instrum. Methods B45 (1990) 6.

[3] G. Amsel, I. Vickridge, Nucl. Instrum. Methods B45 (1990) 12.

[4] Available from the website at http://www.if.ufrgs.br/ pezzi/flatus

[5] M. Tosaki, S. Ito, N. Maeda, Nucl. Instrum. Methods B168 (2000) 543.

[6] M. Tosaki, D. Ohsawa, Y. Isozumi, Nucl. Instrum. Methods B219-220 (2004) 241.

[7] M. Tosaki, D. Ohsawa, Y. Isozumi, Nucl. Instrum. Methods B230 (2005) 59.

[8] J.F. Ziegler, R.F. Lever, and J.K. Hirvonen, in Ion Beam Surface Layer Analysis, eds. O. Meyer, G. Linker and F. Käppeler (Plenum, New York, 1976) Vol. 1 p. 163.

[9] L.R. Doolittle, Nucl. Instr. and Meth. B9 (1985) 344.

[10] M. Mayer, Technical Report IPP9/113, Max-Planck-Institut für Plasmaphysik, Garching, Germany (1997).

[11] N.P. Barradas, C. Jeynes, and R.P. Webb, Appl. Phys. Lett. 71 (1997) 291.

[12] C. Jeynes, N.P. Barradas, P.K. Marriott, G. Boudreault, M. Jenkin, E. Wendler and R.P. Webb, J. Phys. D: Appl. Phys. 36 (2003) R97.

[13] Status of IBA data analysis and simulation software, E. Rauhala, N. P. Barradas, S. Fazinic, M. Mayer, E. Szilágyi, M. Thompson, Nucl. Instrum. Methods Phys. Res. B., accepted

[14] Available from the SigmaCalc website at www.ionbeamcentre.com/sigmacalc

[15] M.J.F. Healy, A.F. Gurbich, Nucl. Instrum. Methods B161-163 (2000) 136.

[16] A.F. Gurbich, Nucl. Instrum. Methods B136-138 (1998) 60.

[17] A.F. Gurbich, Nucl. Instrum. Methods B152 (1999) 403.

[18] L. Wielopolski and R.P. Gardner, Nucl. Instr. Meth. 133 (1976) 303.

[19] N.P. Barradas, Nucl. Instr. and Meth. B225 (2004) 318.

[20] C. Tschalär, Nucl. Instr. Meth. 61 (1968) 141.

[21] C. Tschalär, Nucl. Instr. and Meth. 64 (1968) 237. 
[22] C. Tschalär, H.D. Maccabee, Phys.Rev. B1 (1970) 2863.

[23] E. Szilágyi, F. Pászti, and G. Amsel, Nucl. Instrum. Methods B 100 (1995) 103.

[24] E. Szilágyi, Nucl. Instrum. Methods B 161-163 (2000) 37.

[25] G. Amsel, G. Battistig, A. L’Hoir, Nucl. Instrum. Methods Phys. Res. B 201 (2003) 325.

[26] E. Szilágyi, F. Pászti, Nucl. Instrum. Methods B 85 (1994) 616.

[27] N.P. Barradas, C. Jeynes, O.A. Mironov, P.J. Phillips, and E.H.C. Parker, Nucl. Instrum. and Methods B139 (1998) 239-243.

[28] N.P. Barradas, J.C. Soares, M.F. da Silva, F. Pászti, and E. Szilágyi, Nucl. Instrum. Methods B 94 (1994) 266.

[29] R. G. Elliman, H. Timmers, G. R. Palmer, T. R. Ophel Nucl. Instrum. Methods B 136138 (1998) 649.

[30] N. P. Barradas, J. Phys. D: Appl. Phys. 34 (2001) 2109.

[31] N. P. Barradas, E Alves, S. Pereira, V. V. Shvartsman, A. L. Kholkin, E. Pereira, K. P. O’Donnell, C. Liu, C. J. Deatcher I. M. Watson, M. Mayer, Nucl. Instrum. Methods Phys. Res. B 217 (2004) 479

[32] C.J. Tavares, L. Rebouta, E. Alves, N. P. Barradas, J. Pacaud, J. P. Riviére, Nucl. Instrum. Methods Phys. Res. B188 (2002) 90

[33] N. P. Barradas, E. Alves, D. Babonneau, Nucl. Instrum. Methods Phys. Res. B 219-220 (2004) 919

[34] J.P. Stoquert, T. Szörenyi, Phys. Rev. B 66 (2002) 144108.

[35] R. Irmscher, Ch. Buchal, B. Stritzker, Nucl. Instrum. Methods B51 (1990) 442.

[36] A. Simon, C. Jeynes, R.P. Webb, R. Finnis, Z. Tabatabian, P.J. Sellin, M.B.H. Breese, D.F. Fellows, R. van den Broek, R.M. Gwilliam, Nucl. Instrum. Methods Phys. Res. B 219-220 (2004) 405-409.

[37] www.ee.surrey.ac.uk/ibc/ndf 


\section{Figure Captions}

Figure 1. Cross section $\sigma$, and effective cross section $\sigma^{\mathrm{t}}$ for 1.8, 2.0 and 2.2 $\mathrm{MeV}$ protons for a $160^{\circ}$ scattering angle and normal incidence, as a function of the energy before interaction in a carbon target.

Figure 2. Difference in average energy after interaction due to the energy spread before interaction, for 1.8 and $2 \mathrm{MeV}$ protons for a $160^{\circ}$ scattering angle and normal incidence, as a function of the energy before interaction in a carbon target. The vertical dotted line marks the resonance energy.

Figure 3. Results for 5.05, 5.5 and 6.0 MeV protons in HOPG carbon for a $179.2^{\circ}$ scattering angle and normal incidence. Simulations with the model developed in this work (solid lines) are compared with full double integral calculations (dashed lines). Resonances P1, P2 and P3, at energies 4.8, 5.37 and 5.89 $\mathrm{MeV}$ are observed.

Figure 4. Results for 1.8 and $2 \mathrm{MeV}$ protons in glassy carbon for a $160^{\circ}$ scattering angle and normal incidence. Calculations including both the effect of effective cross section and average energy after interaction (solid lines), only the effect of effective cross section (dashed-dotted line for $2 \mathrm{MeV}$ ) and no effect (dashed lines) are shown.

Figure 5. Results for $2 \mathrm{MeV}$ protons in pirolytic graphite, measured at $140^{\circ}$ scattering angle and near-normal incidence $\left(5^{\circ}\right)$, before and after polishing the sample surface. 
Figure 6. Results for $2 \mathrm{MeV}$ protons in a graphite rod, measured at $140^{\circ}$ scattering angle and near-normal incidence $\left(5^{\circ}\right)$.

Figure 7. Results for $2 \mathrm{MeV}$ protons in glassy carbon for a $160^{\circ}$ scattering angle and different incidence angles. The vertical dashed line indicates the resonance maximum at $0^{\circ}$.

Figure 8. Results for $2 \mathrm{MeV}$ protons in $\mathrm{c}-\mathrm{Si}$, measured at near-normal incidence $\left(3^{\circ}\right)$ and grazing incidence $\left(75^{\circ}\right)$.

Figure 9. Results for the Mylar $1 \mu \mathrm{m} / \mathrm{Ni} 4.2 \mu \mathrm{m}$ sample at different proton energies. For 2025 and $2066 \mathrm{keV}$ the results obtained not taking the resonance effect into account are also shown (dashed lines).

Figure 10. Integrated $\mathrm{C}$ yield for the Mylar $1 \mu \mathrm{m} / \mathrm{Ni} 4.2 \mu \mathrm{m}$ sample as a function of proton beam energy. The data (squares, solid line) and calculated values with (circles) and without (triangles) the resonance effect are shown. 
Fig. 1

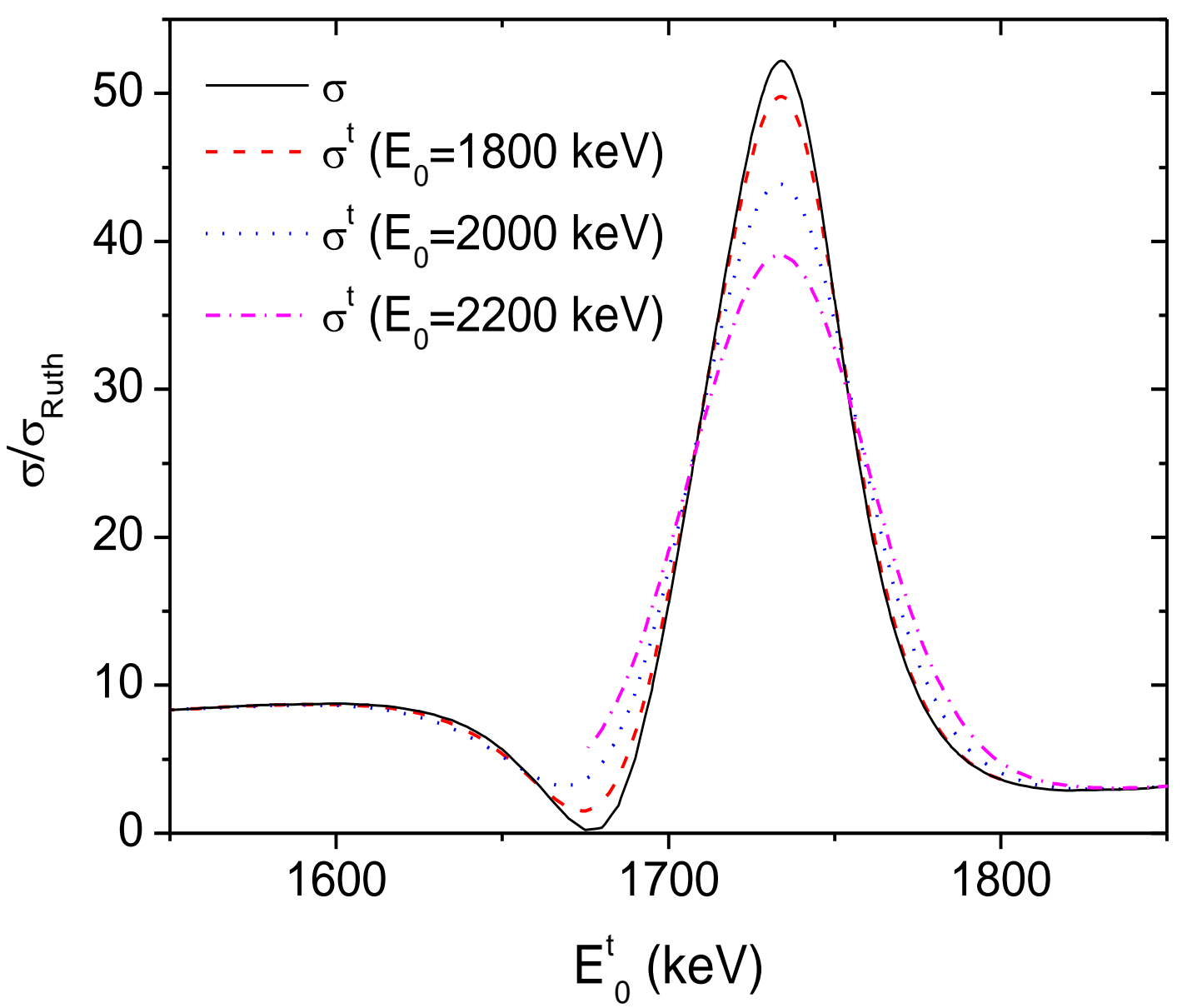


Fig. 2

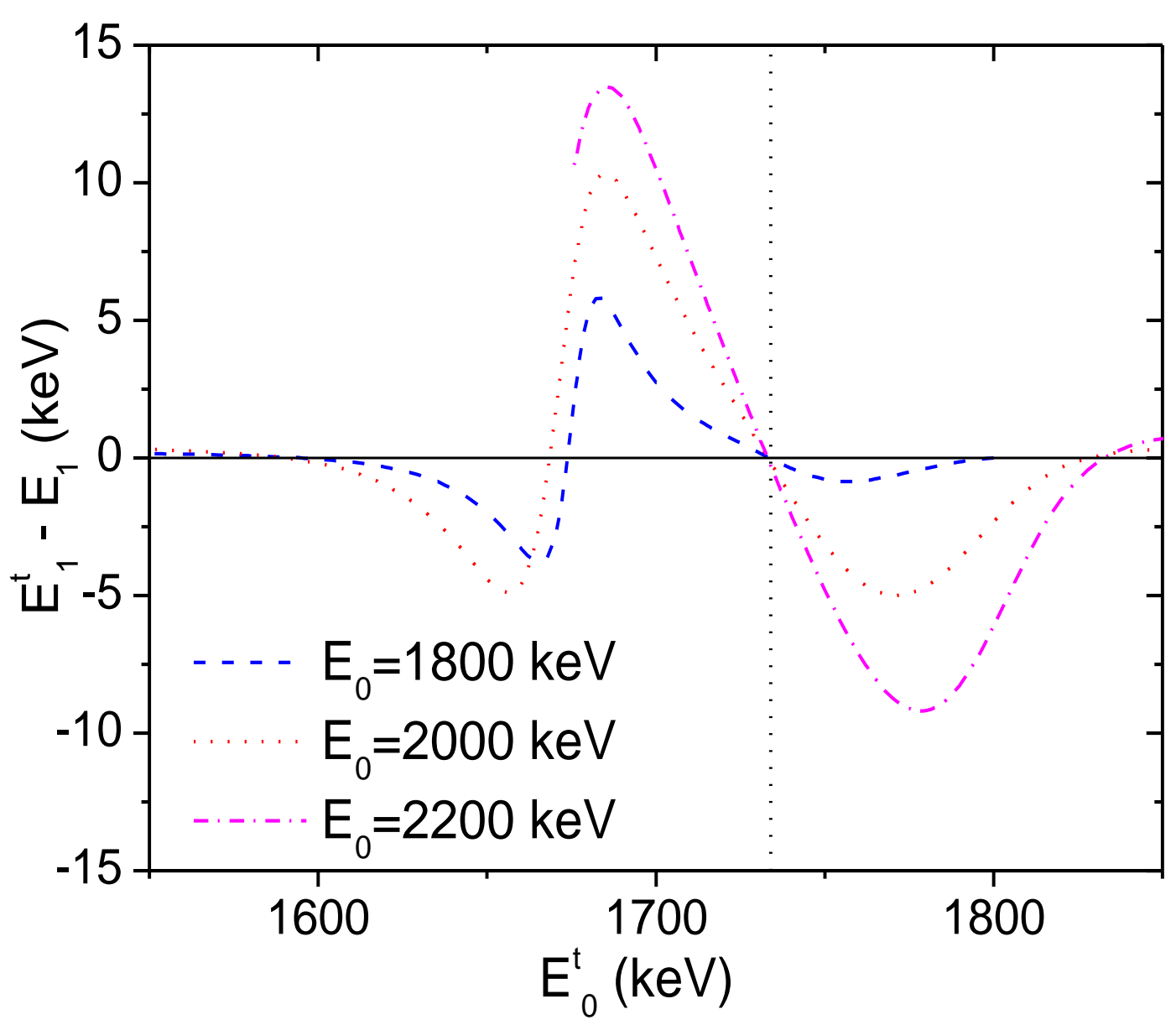


Fig. 3

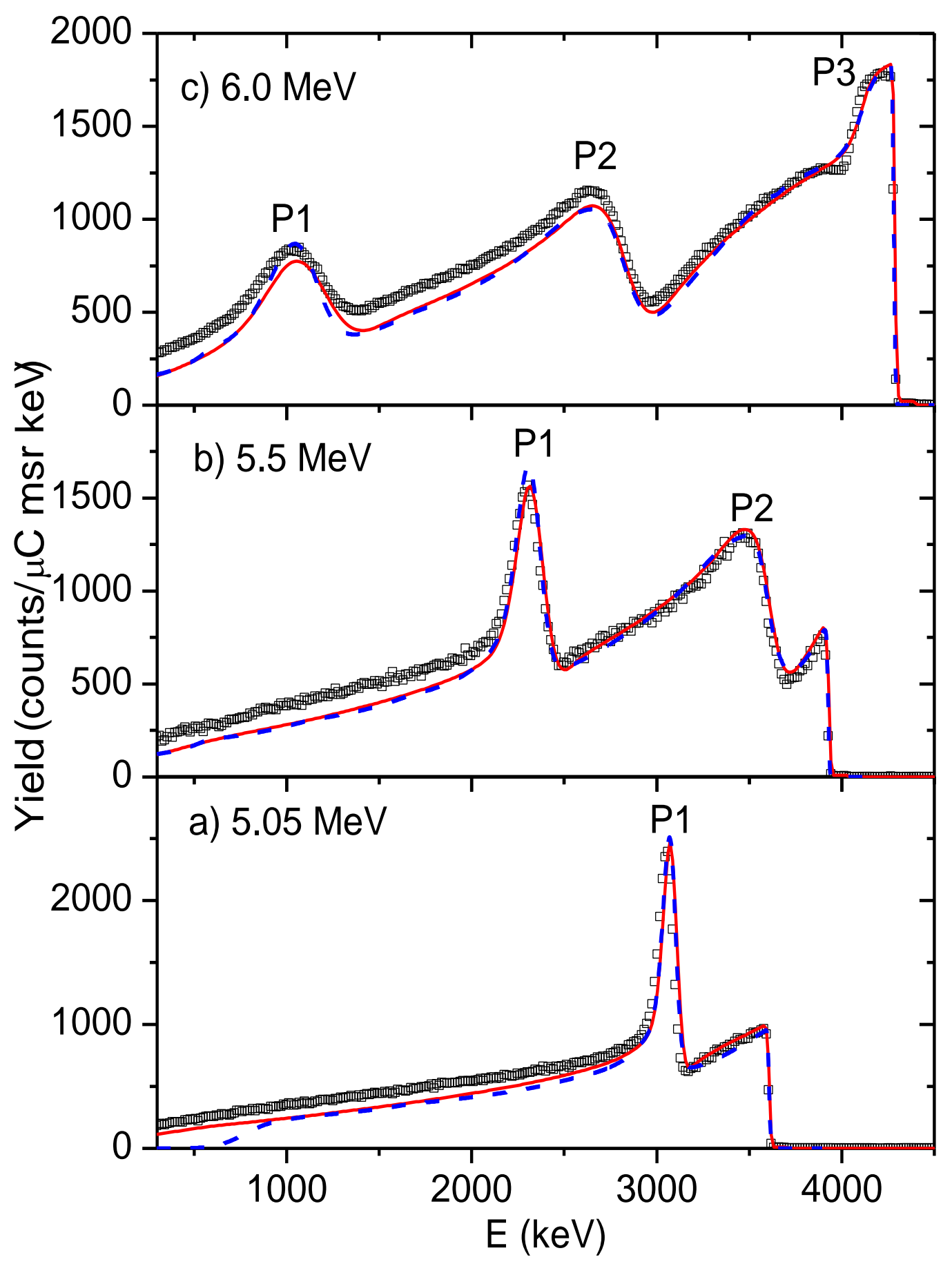


Fig. 4
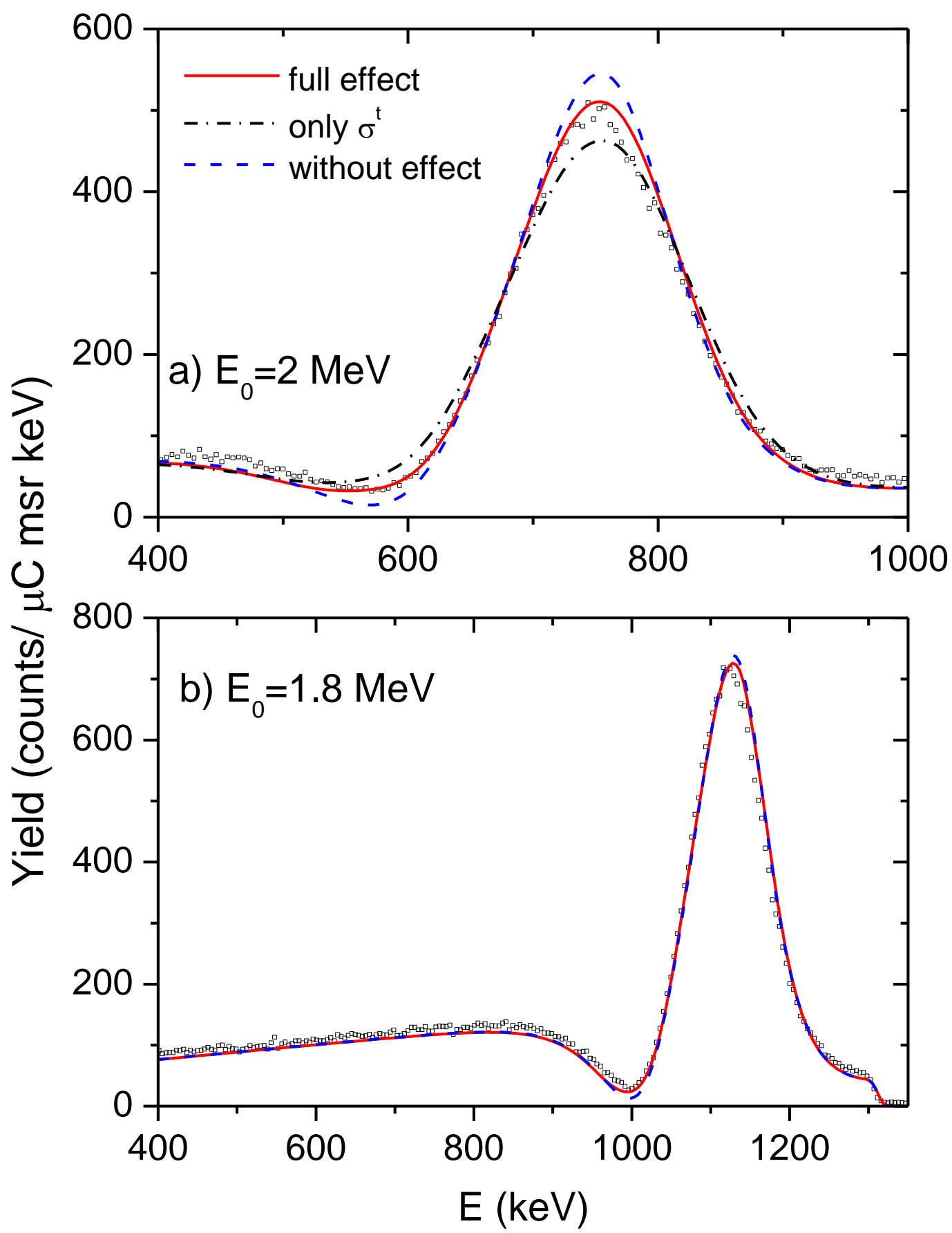
Fig. 5

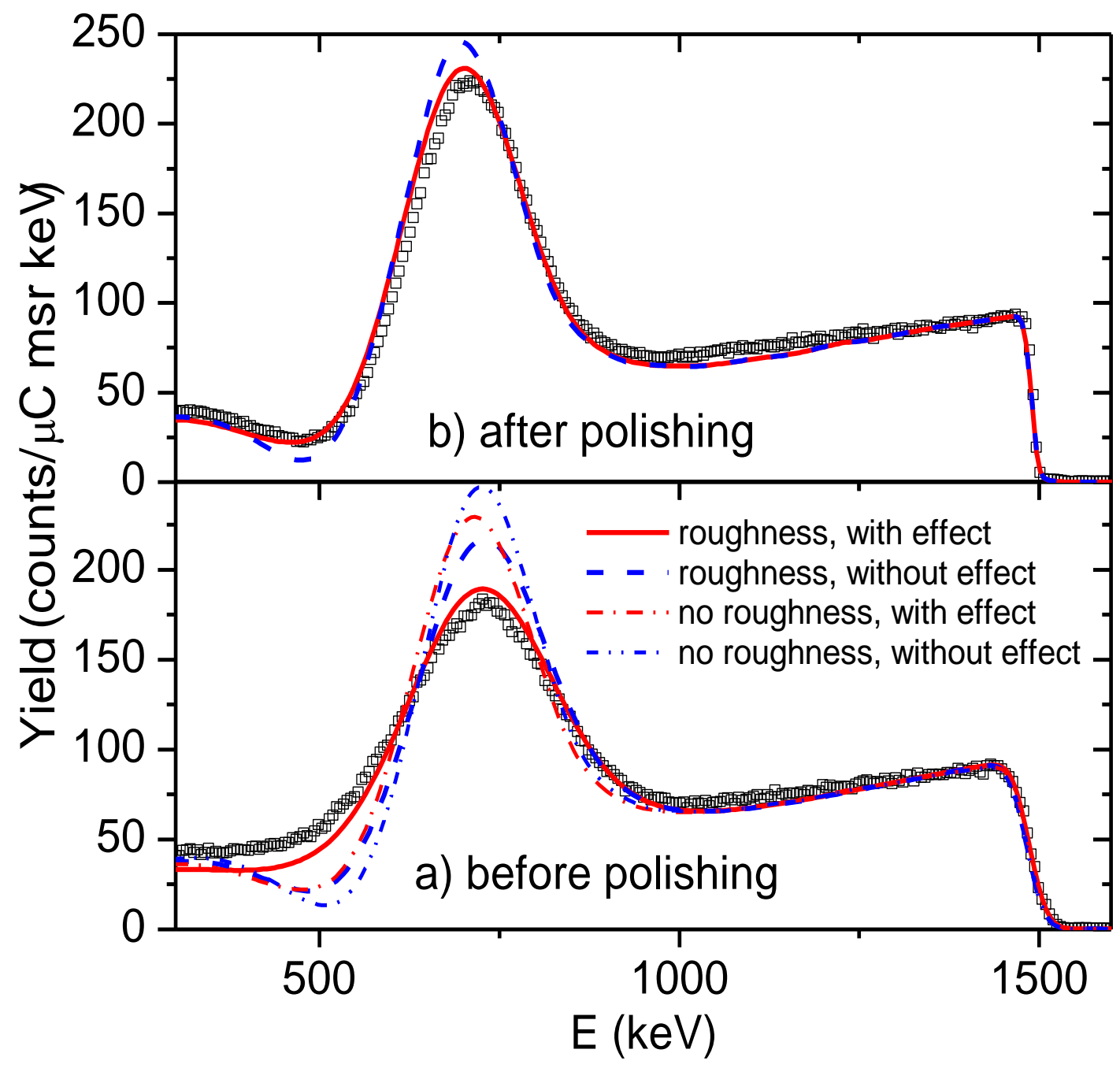


Fig. 6

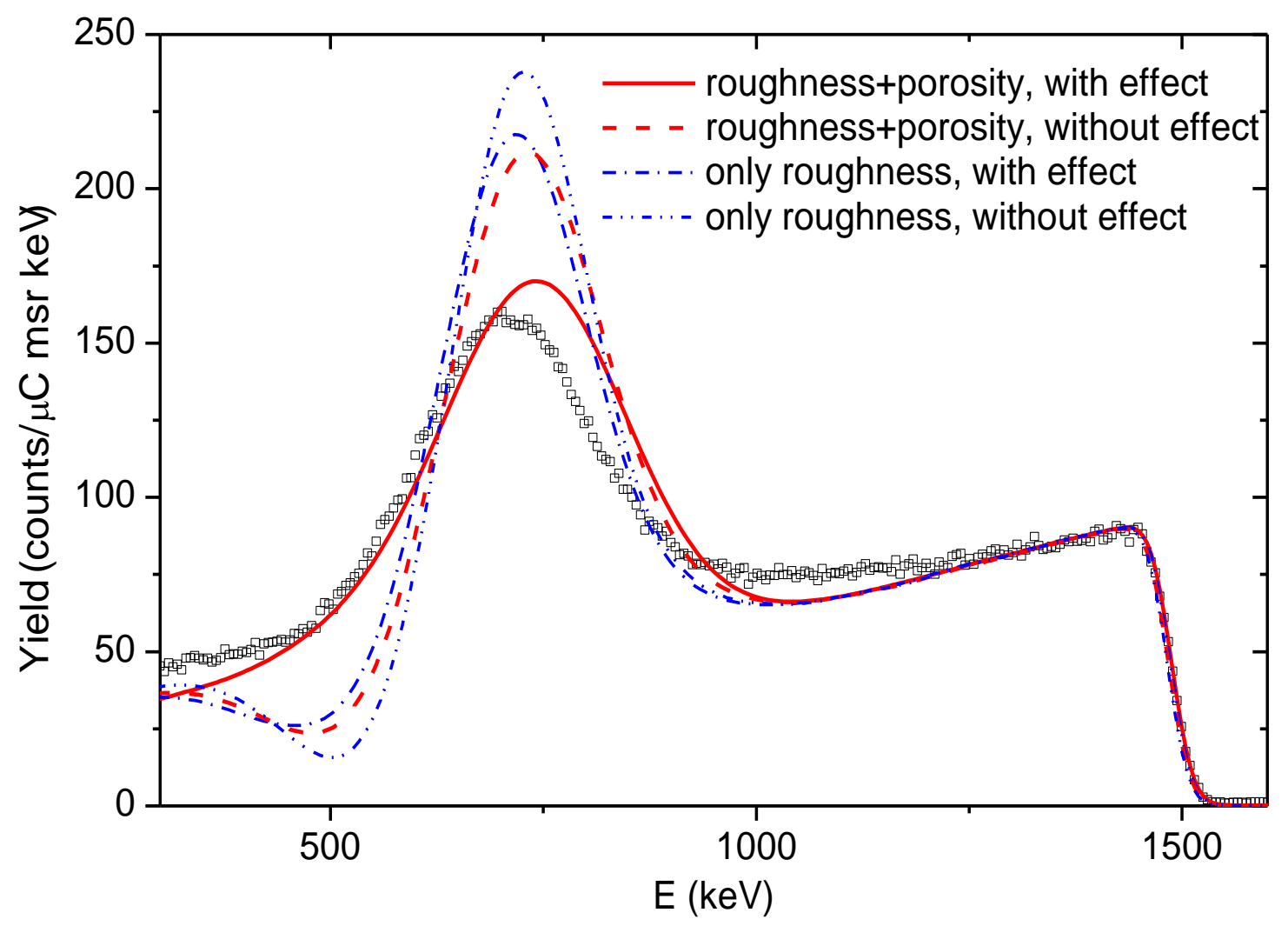


Fig. 7

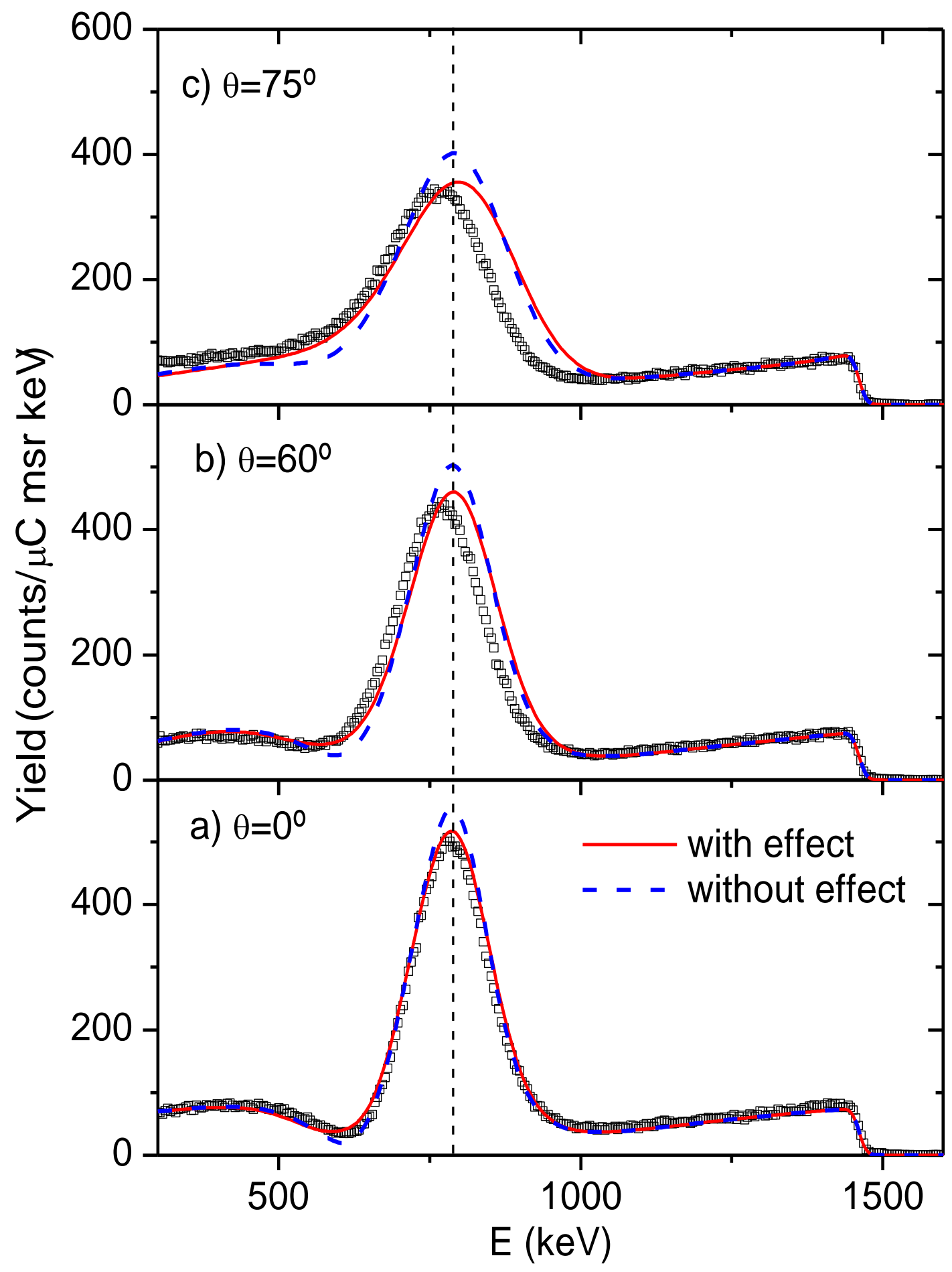


Fig. 8

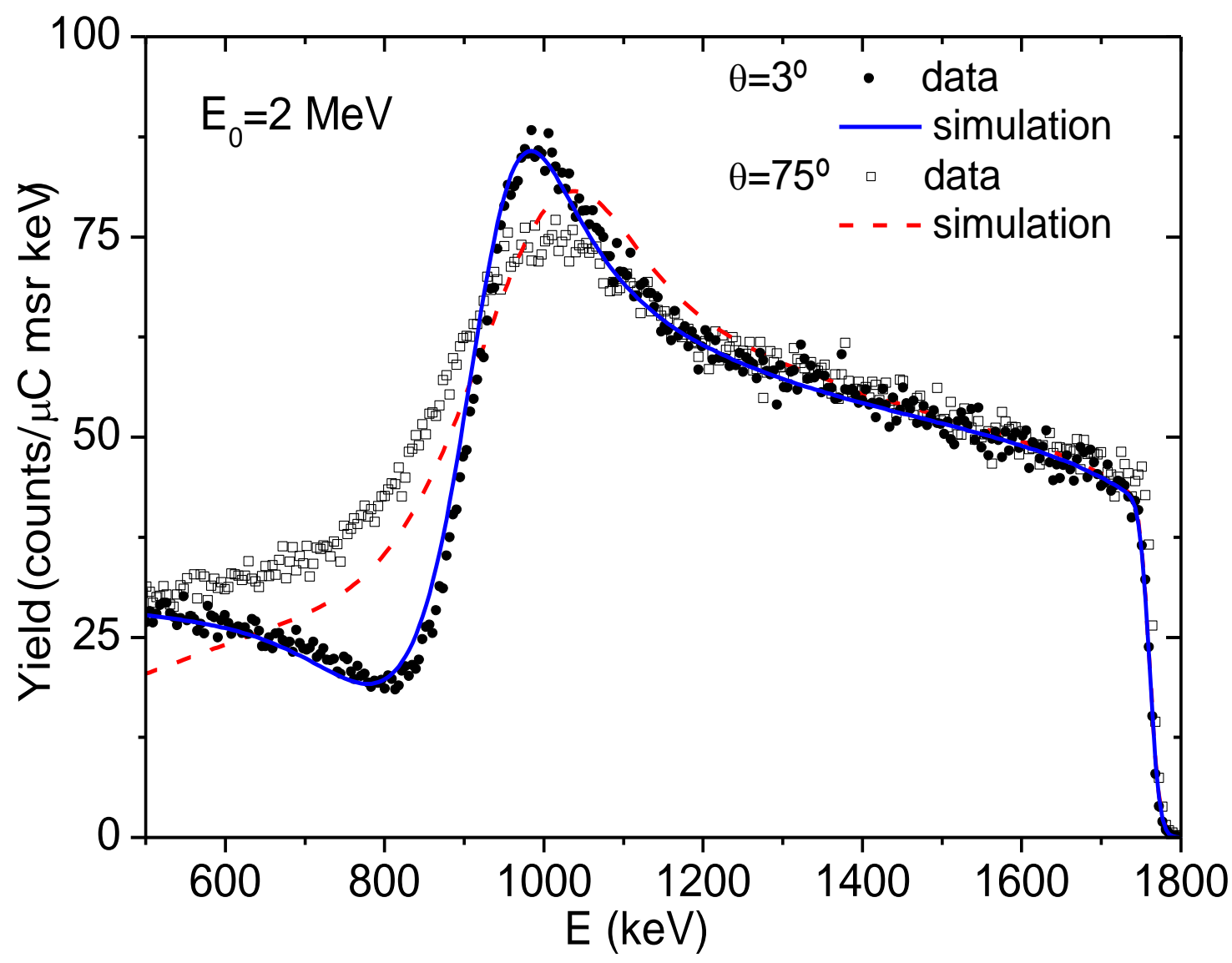


Fig. 9

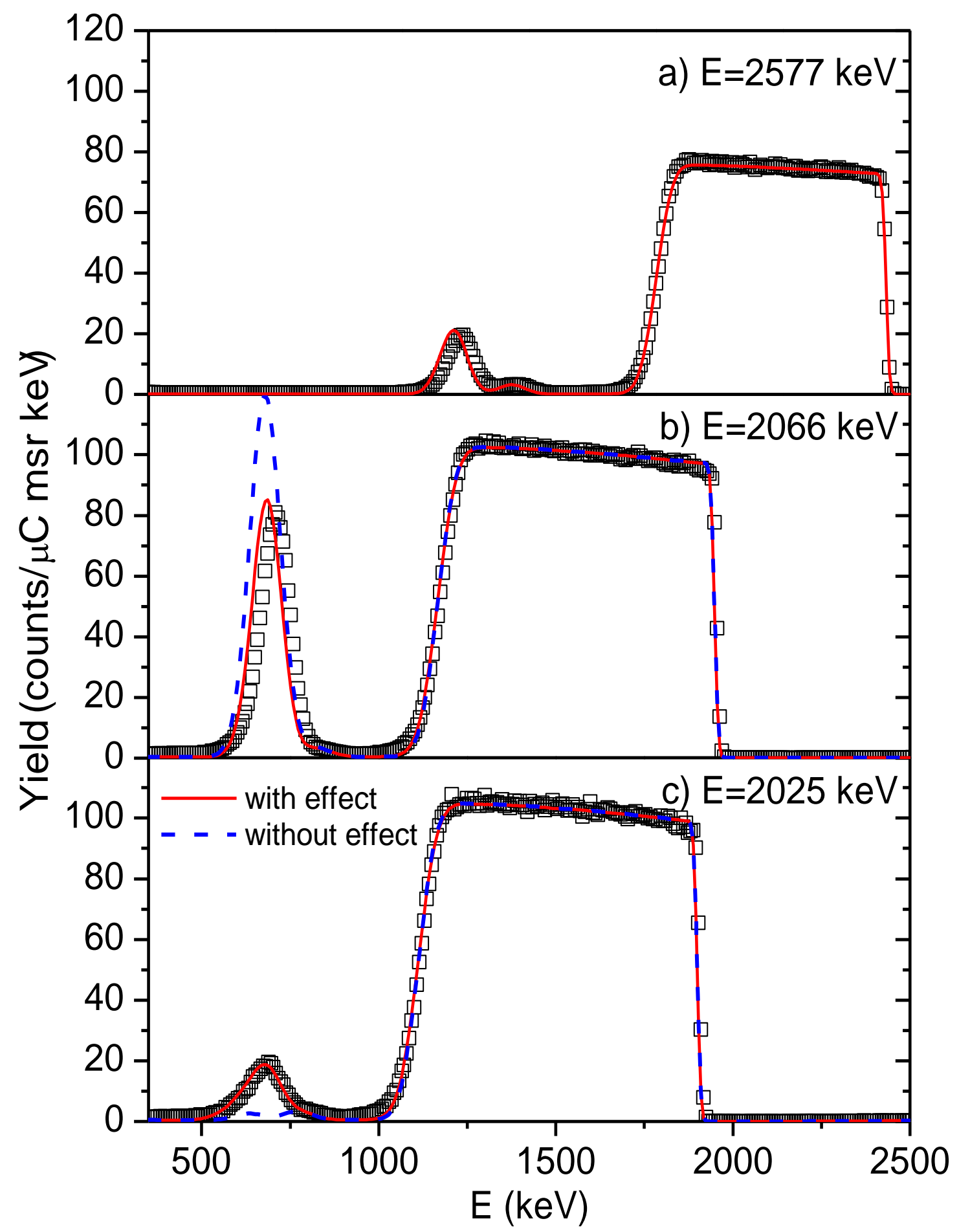


Fig. 10

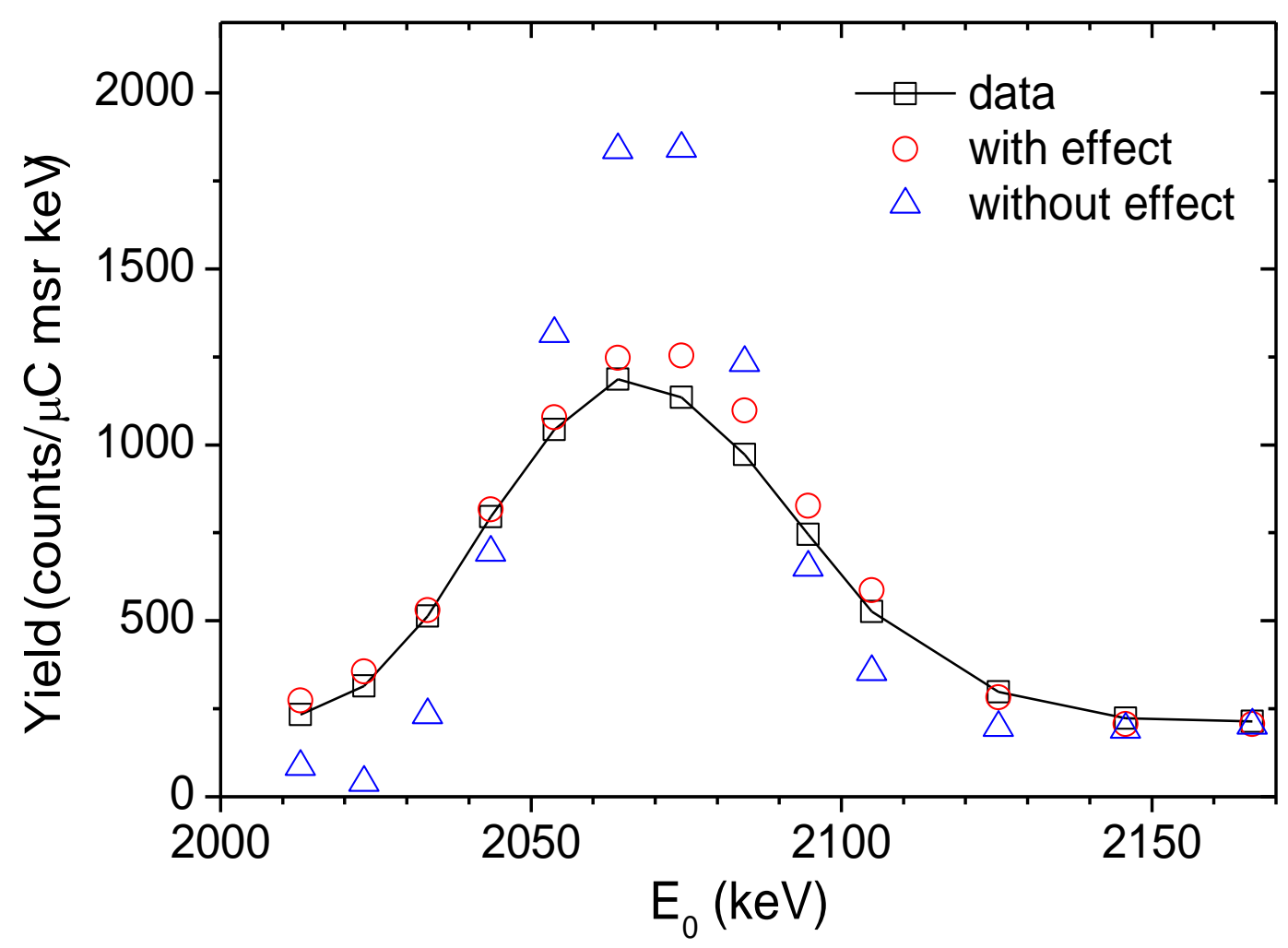

